\section{BMJ Open Sport \& Exercise Medicine}

\title{
Cardiac abnormalities in athletes after SARS-CoV-2 infection: a systematic review
}

\section{Juliette C van Hattum (1D , Jessica L Spies, ${ }^{1}$ Sjoerd M Verwijs, ${ }^{1}$} Germaine C Verwoert, ${ }^{2}$ R Nils Planken, ${ }^{3}$ S Matthijs Boekholdt, ${ }^{1}$ Maarten Groenink, ${ }^{1}$ Arjan Malekzadeh, ${ }^{4}$ Yigal M Pinto, ${ }^{1}$ Arthur A M Wilde, ${ }^{1}$ Harald T Jorstad ${ }^{1}{ }^{1}$

To cite: van Hattum JC, Spies JL, Verwijs SM, et al. Cardiac abnormalities in athletes after SARS-CoV-2 infection: a systematic review. BMJ Open Sport \& Exercise Medicine 2021;7:e001164. doi:10.1136/ bmjsem-2021-001164

- Additional supplemental material is published online only. To view, please visit the journal online (http://dx.doi. org/10.1136/bmjsem-2021001164).

Accepted 26 September 2021

Check for updates

\section{(C) Author(s) (or their} employer(s)) 2021. Re-use permitted under CC BY-NC. No commercial re-use. See rights and permissions. Published by BMJ.

${ }^{1}$ Department of Cardiology, Amsterdam UMC, Universtiy of Amsterdam, Amsterdam, The Netherlands

${ }^{2}$ Department of Cardiology, Erasmus University Rotterdam, Rotterdam, The Netherlands ${ }^{3}$ Department of Radiology, Amsterdam UMC, University of Amsterdam, Amsterdam, The Netherlands

${ }^{4}$ Department of Medical Library, Amsterdam UMC, University of Amsterdam, Amsterdam, The Netherlands

\section{Correspondence to} MD Juliette $C$ van Hattum; j.c.vanhattum@amsterdamumc. $\mathrm{nl}$

\section{ABSTRACT}

Objectives Quantification of pericardial/myocardial involvement and risks of sudden cardiac arrest/sudden cardiac death (SCA/SCD) after SARS-CoV-2 infection in athletes who return to sports.

Design Systematic review on post-SARS-CoV-2 infection pericardial/myocardial manifestations in athletes.

Data sources Combinations of key terms in Medline, Embase and Scopus (through 2 June 2021).

Eligibility criteria for selecting studies Inclusion: athletes, with cardiovascular magnetic resonance (CMR) or echocardiography after recovery from SARS-CoV-2 infection, including arrhythmia outcomes. Exclusion: study population $\geq 1$ individual comorbidity and mean age $<18$ or $>64$ years. Quality assessment was performed using Joanna Briggs Institute Critical Appraisal tools checklists. Results In total, 12 manuscripts (1650 papers reviewed) comprising 3131 athletes (2198 college/ student athletes, 879 professional athletes and 54 elite athletes) were included. The prevalence of myocarditis on echocardiography and/or CMR was $0 \%-15 \%$, pericardial effusion $0 \%-58 \%$ and late gadolinium enhancement (LGE) $0 \%-46 \%$. Weighted means of diagnosed myocarditis were $2.1 \%$ in college/student athletes and $0 \%$ in elite athletes. The prevalence of LGE was markedly lower in studies with high-quality assessment scores $(3 \%-4 \%)$ versus low scores (38\%-42\%). A single study reported reversibility of myocardial involvement in $40.7 \%$. No important arrhythmias were reported. Ten studies $(n=4171)$ reporting postrecovery troponin $\mathrm{T} / \mathrm{I}$ found no clear relationship with cardiac abnormalities.

Summary/conclusion Athletes have an overall low risk of SARS-CoV-2 pericardial/myocardial involvement, arrhythmias and SCA/SCD. Rates of pericardial/myocardial abnormalities in athletes are highly variable and dependent on study quality. Troponin screenings seem unreliable to identify athletes at risk for myocardial involvement. Prospective athlete studies, with pre-SARS-CoV-2 imaging (CMR), including structured follow-up and arrhythmia monitoring, are urgently needed.

\section{INTRODUCTION}

SARS-CoV-2 has spread globally and was declared a pandemic by the WHO on 11 March 2020. ${ }^{1}$ Most studies performed early in

\section{Key messages}

What is already known

- Rates of sustained cardiac involvement after SARSCoV-2 recovery have been reported to be highly variable in both symptomatic and asymptomatic athletes.

- Physical exercise is thought to be associated with a worsening of prognosis in viral myocarditis in general.

- Risk of potentially fatal arrhythmias in athletes with cardiac involvement after recovery from SARSCoV-2 is largely unknown.

- No clear consensus exists on how to screen for post- SARS-CoV-2 cardiac abnormalities, and existing consensus documents include different diagnostic modalities (ECG, imaging and biomarkers) and cut-offs.

What are the new findings

- Athletes have an overall low risk $(0 \%-5 \%)$ of SARSCoV-2 pericardial/myocardial involvement, with no reported arrhythmias or sudden cardiac arrest/sudden cardiac death.

- Rates of pericardial/myocardial abnormalities in athletes are highly variable per study and dependent on study quality, with higher quality studies reporting rates of $3 \%-4 \%$.

- Troponin screenings are unreliable to identify athletes at risk for myocardial involvement.

- Prospective athlete studies, with pre-SARS-CoV-2 CMR, including structured follow-up and arrhythmia monitoring, are urgently needed.

the pandemic focused on cardiac complications in hospitalised and severely ill patients, but recently, there has been marked interest in SARS-CoV-2 cardiac complications and sequelae in young and healthy individuals, including athletes. ${ }^{2}$

The initial studies in asymptomatic athletes or athletes with a mild or moderate course of illness reported considerable sustained cardiac involvement after recovery from SARS-CoV-2 infection. Later studies have not replicated these findings. Considering that 
physical exercise has been associated with a worsening of prognosis in viral myocarditis, ${ }^{3-5}$ cardiac involvement in athletes who have recovered from SARS-CoV-2 infection is of particular relevance, and a clear scope of the problem is needed.

Multiple return-to-play consensus statements have been published to assist clinicians in screening for SARS-CoV-2 cardiac abnormalities. ${ }^{6-9}$ These consensus statements are likewise highly variable and include different diagnostic modalities (eg, ECGs, exercise tests, Holter monitoring, troponin levels and echocardiography) and cut-offs. Numerous statements place emphasis on serum troponin levels, as they have been observed to be elevated in severely ill patients as compared with those with milder COVID$19^{10}$ and negatively correlated with prognosis. ${ }^{11-14}$ Yet, little is known about the efficacy of such screenings. ${ }^{15} 16$ Finally, the risk of potentially fatal arrhythmias in athletes with cardiac involvement after recovery from SARS-CoV-2 remains largely unknown.

We therefore aimed to address the following research questions: in athletes recovered form SARS-CoV-2 infection: (1) what is the prevalence of SARS-CoV-2 cardiac involvement (as found using imaging investigations), stratified by study quality?; (2) what is the incidence of ventricular tachyarrhythmia's (and SCA/SCD) in athletes with documented cardiac involvement?

We performed a systematic search, review and quality assessment to provide a comprehensive overview of myocardial and pericardial involvement after SARS-CoV-2 infection, long-term cardiac sequelae after infection and risks of sudden cardiac arrest/sudden cardiac death (SCA/SCD) in athletes.

\section{METHODS}

\section{Literature search}

We conducted a systematic search strategy according to Preferred Reporting Items for Systematic Reviews and Meta-Analyses guidelines $^{17}$ (PROSPERO ID:CRD42021233964). The comprehensive electronic search was performed in Medline (Ovid), Embase (Ovid) and Scopus (final update 2 June 2021), with combined terms or synonyms for: COVID-19, SARS-CoV-2, cardiovascular imaging, cardiac MRI, echocardiography, SCD, athletes, (young) adults and arrhythmias (figure 1).
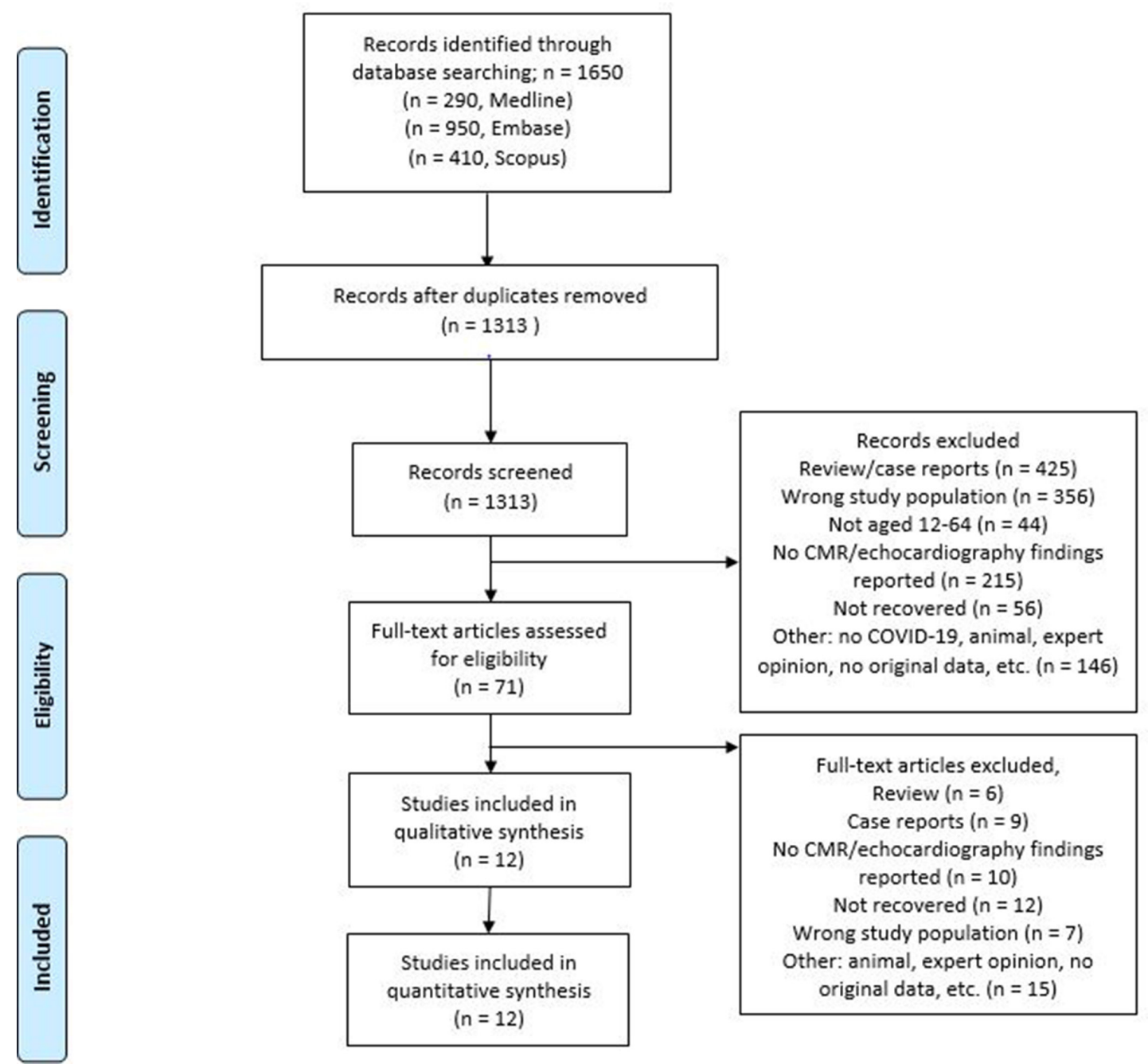

Figure 1 PRISMA 2009 flow chart describing selection of studies included in the systematic review. ${ }^{17} \mathrm{CMR}$, cardiovascular magnetic resonance; PRISMA, Preferred Reporting Items for Systematic Reviews and Meta-Analyses. 
Conference abstracts from Embase (Ovid) were included to identify unpublished studies. Subsequently, we performed manual citation and reference searching of the included studies in Google Scholar. The full search strategy can be found in online supplemental file 1 .

\section{Study selection}

We included studies when they: 1) contained clinical data on athletes recovered from SARS-CoV-2 infection, (2) reported cardiovascular imaging by either cardiovascular magnetic resonance (CMR) or echocardiography, (3) performed investigations after SARS-CoV-2 infection, with emphasis on arrhythmia outcomes. Athletes were defined as individuals of young or adult age, either amateur or professional, who engage in regular exercise training and participate in official sports competitions. College/student athletes were defined as individuals of young age, who participate in organised competitive sports sponsored by the educational institution in which the student is enrolled, and elite athletes, (national team, Olympians, and professional athletes) were defined as athletes who generally exercise $>10$ hours/ week. ${ }^{18}$ Exclusion criteria were: (1) study population with $\geq 1$ mean individual comorbidities, (2) mean age of total study population $<18$ or $>64$ years, (3) no original data reported and (4) case reports. Two investigators (JCvH and JLS) independently screened and assessed all identified manuscripts. Discrepancies were resolved by discussion between the two reviewers and external teambased expert ${ }^{19}$ opinion, until consensus was reached.

\section{Outcomes}

Our primary outcome of interest was myocardial and/or pericardial abnormalities/manifestations as documented using CMR or echocardiography, in athletes recovered from SARS-CoV-2 infection. Secondary outcomes included arrhythmias (if reported) and SCA/SCD and potential associations between imaging abnormalities and serum troponin levels.

\section{Data extraction}

First, we extracted data on authors, demographic findings, sample sizes, time to imaging, clinical features, elevated troponin levels (according to individual studydefined cut-offs), reported arrhythmias or SCA/SCD and time of investigation/duration of follow-up (FU). Second, we extracted data on CMR findings, which included rates of elevated $\mathrm{T} 1$ time and elevated $\mathrm{T} 2$ time. In short, both $\mathrm{T} 1$ and $\mathrm{T} 2$ times are calculated using an MRI technique for myocardial tissue characterisation. An increased $\mathrm{T} 1$ time can be indicative for cardiomyopathies and increased T2 times for myocardial inflammation. We also extracted data of pericardial effusion (PE) according to individual study-defined cut-offs, pericardial enhancement and presence of late gadolinium enhancement (LGE). In short, LGE demonstrated regional differences in myocardial extracellular volume, and abnormal uptake and washout patterns (myocardial, non-ischaemic, pericardial, hingepoint or other) within the extracellular space, such as seen in myocardial inflammation and fibrosis. In addition, we extracted the criteria used to diagnose myocarditis (if reported) and the amount of diagnosed myocarditis. Third, we extracted data on echocardiographic findings, which included left ventricular (LV) end-diastolic diameter (EDD), LV ejection fraction (LVEF), right ventricle (RV) ejection fraction (RVEF), right ventricle function/tricuspid annular plane systolic excursion (TAPSE) (ie, right ventricular longitudinal contraction or shortening, a measure of right ventricular function), LV and RV global longitudinal strain (GLS) (ventricular deformation patterns), early to late diastolic transmitral flow velocity (E/A), ratio of early diastolic mitral inflow velocity/ tissue velocity $\left(\mathrm{E} / \mathrm{e}^{\prime}\right)$, segmental wall motion abnormalities, PE (according to study-defined cut-offs) and systolic pulmonary artery pressure. Two independent reviewers extracted the relevant data, which was stored in a Microsoft Excel database and exported to $\mathrm{R}$ for statistical analysis.

\section{Statistical methods and quality assessment}

Data are presented as means and SD, medians with IQR and weighted means (for data synthesis), as appropriate. ${ }^{20}$ Statistical analyses were performed using R (V.1.3.1993).

Data quality was assessed using the Joanna Briggs Institute Critical Appraisal tools checklists ${ }^{21}$ by two authors independently. Discrepancies were resolved after joint article review and discussion. The relationship between data quality and severe cardiac abnormalities, defined as LGE and PE on CMR, was subsequently plotted.

\section{RESULTS}

The initial search yielded 1650 papers. After extensive review, we included a total of 12 manuscripts comprising 3131 recovered post-SARS-CoV-2 athletes. Except for the study by Gervasi et $a l^{22}$ all studies were cross-sectional or retrospective and did not include pre-SARS-CoV-2 imaging. Study and demographic characteristics of the respective studies are shown in table 1 for studies using CMR as their primary imaging modality, and in table 2 for studies using echocardiography as their primary imaging modality. Overall, median age of all study participants was 22years (IQR 18-41); 36\% were women. In five studies comprising 268 athletes including study controls CMR was performed; in three studies comprising 897 athletes including study controls echocardiography was performed; in four studies comprising 1968 athletes including study controls both CMR and echocardiography were performed. COVID-19 was diagnosed by real-time reverse transcriptase PCR in seven studies, by antibody testing in two studies and in two studies by one of the two; one study (Cavigli $e t a l^{23}$ ) study did not report diagnostic tests. All included athletes had recovered from SARS-CoV-2 infection, of which $0.1 \%$ recovered in-hospital and $99.9 \%$ at home. Two studies (Gervasi et al and Cavigli et al) reported arrhythmia outcomes, and one study (Moulson $e t a l^{24}$ ) reported a resuscitated SCA after 


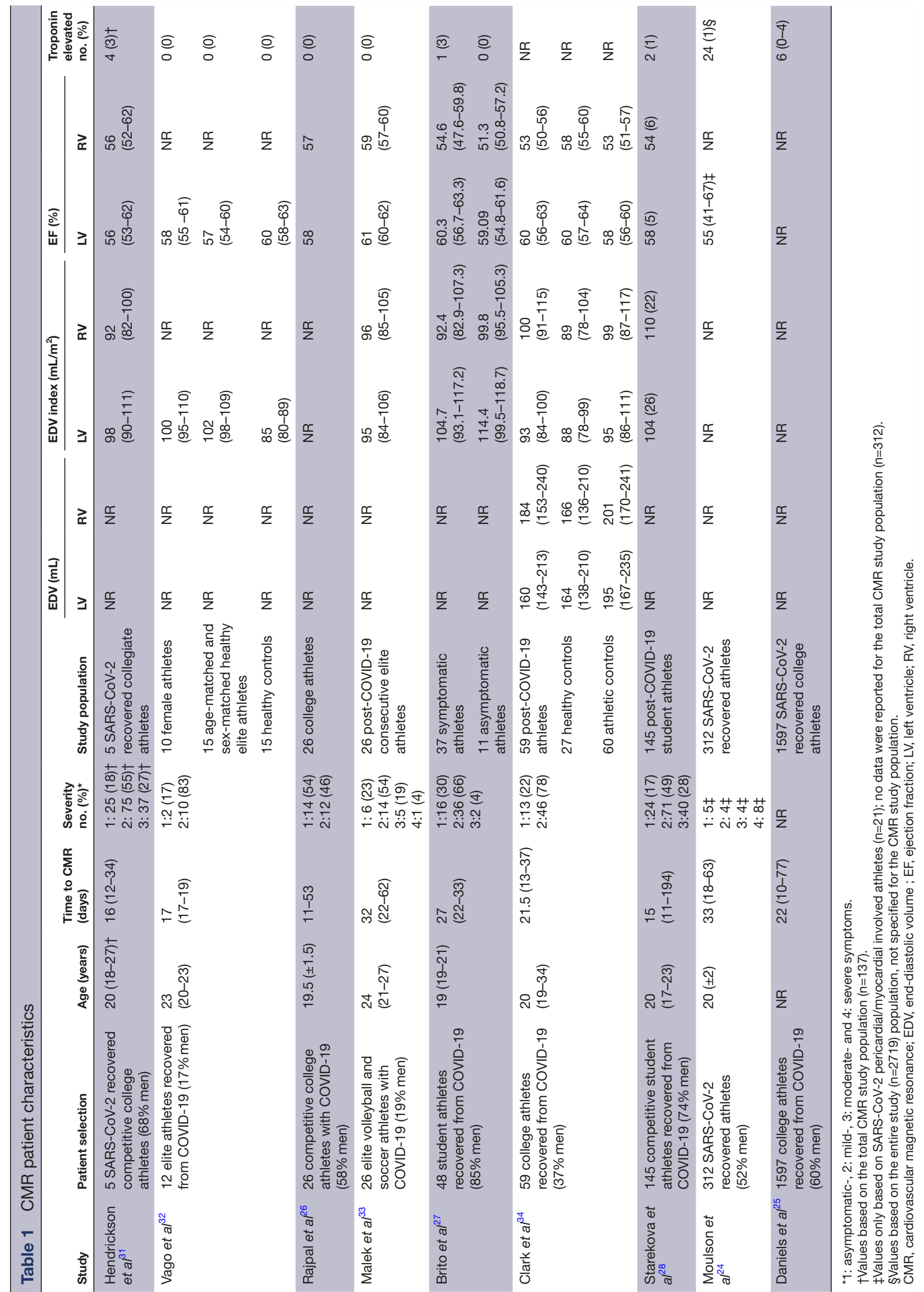




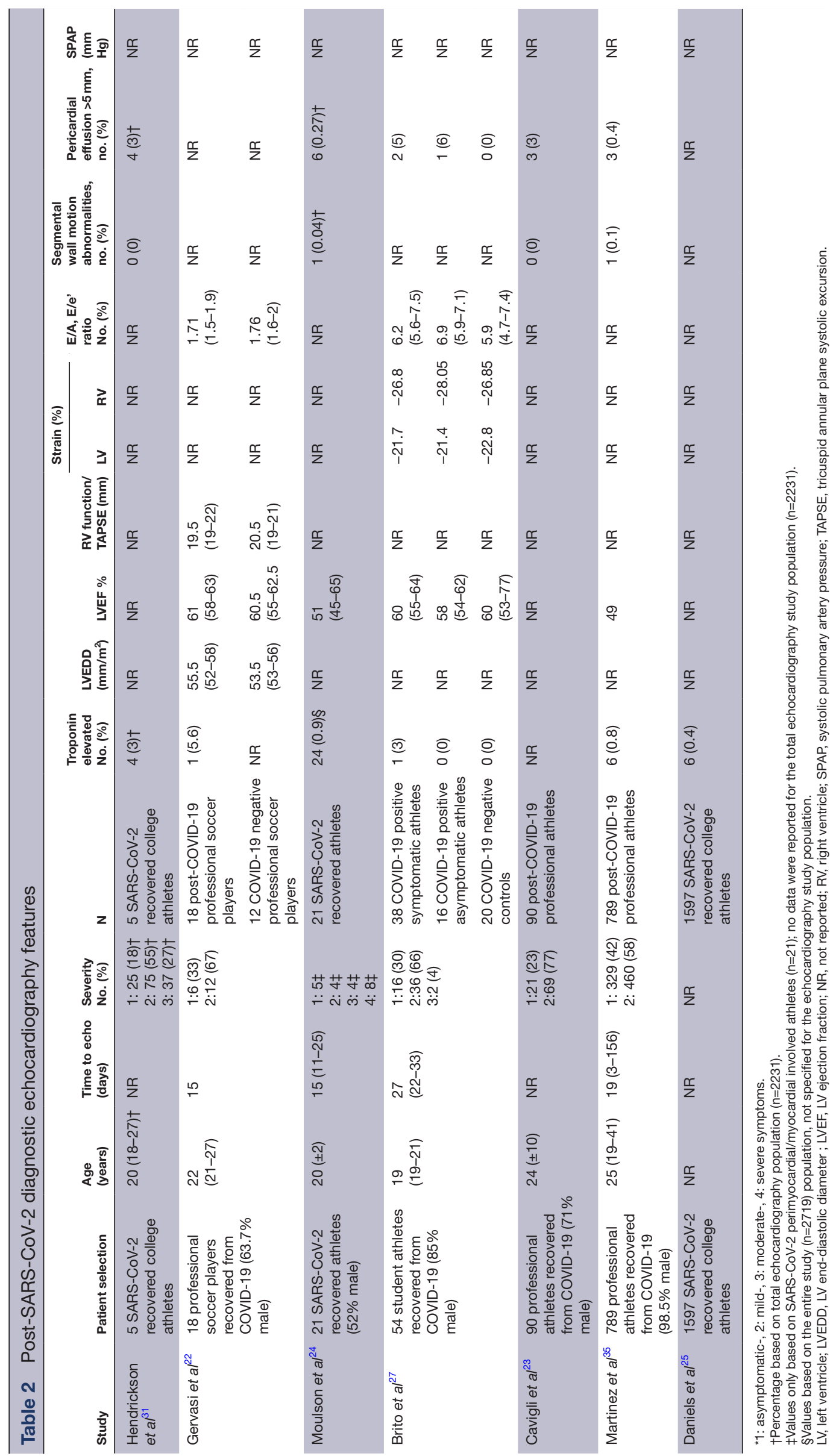


recovery. Only one study (Daniels $e t a l^{25}$ ) reported structured FU.

\section{Risk of bias}

Relevant checklists according to the Joanna Briggs Institute Critical Appraisal tools were applied (eight cohort studies, two cross-sectional studies, one case series and one case-control study). Detailed quality assessment for individual studies can be found in online supplemental file 1 . The risk of bias was the highest in the cohort studies of Rajpal $e t a l^{26}$ and the cross-sectional study of Brito et $a l_{.}^{27}$ The risk of bias was the lowest in the casecontrol study of Gervasi $e t a t^{22}$ and the case-series study of Starekova $e t a l .{ }^{28}$ Other prevalent methodological issues in the cohort studies were not adequately reporting if the participants were free of outcomes at the start of the study $(8 / 8)$, not reporting completion of FU or reasons for incomplete $\mathrm{FU}(7 / 8)$ and not reporting strategies used to address incomplete $\mathrm{FU}(7 / 8)$.

\section{CMR findings}

Studies including CMR are outlined in table 1; main myocardial and pericardial findings are summarised in table 3. Figure 2 illustrates the prevalence of total LGE stratified by risk of bias, and figure 3 illustrates the prevalence of $\mathrm{PE}$ stratified by risk of bias. Median time interval from symptom onset to CMR examination was 32 (IQR 11-194) days. In nine studies in 2477 athletes and study controls, the weighted mean for the prevalence of elevated (according to study-defined cut-offs) T1 was $1.5 \%$, elevated T2 was 2.5\%, LGE including hinge-point fibrosis was $4.9 \%$, LGE excluding hinge-point fibrosis was $2.6 \%$ and PE $11.3 \%$. The weighted mean for the prevalence of active myocarditis according to the Lake Louise criteria (LLC) ${ }^{29}$ or modified LLC ${ }^{30}$ was $1.9 \%$.

\section{Athletes}

In total nine studies reported findings in 2360 athletes (2326 student/college athlete, and 34 elite athletes), whereof $22 \%$ were asymptomatic, $59 \%$ had mild, $19 \%$ had moderate and $0.2 \%$ severe symptoms. The weighted overall mean for the prevalence of elevated $\mathrm{T} 1$ was $1.5 \%$, T2 2.6\%, LGE 5.4\% (six studies reported only total LGE with no specification whether this was myocardial or pericardial), LGE excluding hinge-point fibrosis 2.6\%, PE $11.3 \%$, myocardial LGE (if reported) $1.9 \%$ and pericardial LGE (if reported) 18.7\%. Overall, $2.0 \%$ met the LLC for myocarditis, of which $2.1 \%$ in college/student athletes, and $0 \%$ in elite athletes. Daniels $e t$ al and Starekova $e t$ al reported both one athlete with pericarditis, and Moulson et al reported one athlete with peri-myocarditis. We observed considerable differences between individual studies, ranging from no myocardial involvement (Vago et al) to $15 \%$ (Rajpal et $a l^{26}$ ). Hendrickson et $a l^{31}$ reported CMR findings in only five athletes, with no abnormal findings detected by CMR.

Most studies reported the presence of PE using a cutoff of $10 \mathrm{~mm}$; Brito et $a l^{27}$ applied $5 \mathrm{~mm}$ as their cut-off.
Consequently, Brito et al reported (in college athletes) the highest prevalence of PE $(58 \%)$. Of note, they also reported significantly higher rates of pericardial LGE in asymptomatic athletes as compared with symptomatic athletes $(82 \%$ vs $27 \%, \mathrm{p}=0.003)$. In elite athletes specifically, Vago $e t a l^{32}$ reported no abnormal T1, T2 or LGE, or myocarditis (LLC). Also in elite athletes, Malek $e t a l^{33}$ reported no abnormal T1 but elevated T2 in 4\%, LGE in $4 \%$ and PE in $8 \%$. Moulson et al only reported CMR values of 21 athletes with pericardial/myocardial involvement and not in the total CMR population $(n=312)$. The largest (survey) study (Daniels $e t a l^{25}$ ) reported that 37 $(2.3 \%)$ athletes met diagnostic criteria for myocarditis. Interestingly, these $37 \mathrm{CMR}$-identified subclinical potential cases of myocarditis included 20 athletes without cardiovascular symptoms and with normal ECGs, echocardiography and troponin test results. When compared with alternative diagnostic strategies, the CMR strategy resulted in a 7.4-fold diagnostic increase compared with the symptom driven strategy, and a 2.8-fold increase compared with the ECG, echocardiogram and troponin strategy. In addition, the survey study by Daniels $e t a l^{25}$ was the only study to report FU CMRs in $73 \%$ of individuals with clinical or subclinical myocarditis, with the second CMR taking place between 4 and 14 weeks after confirmation of SARS-CoV-2 infection. Complete resolution of both T2 mapping and LGE was found in $40.7 \%$ of these individuals; resolution of T2 mapping abnormalities but persistent LGE was found in 59.3\%. No other FU data, such as results from arrhythmia monitoring, was reported in this study.

\section{Study controls (healthy and/or athletes)}

In total, two studies reported findings in 117 controls (15 age-matched and sex-matched elite athletes, 42 healthy controls and 60 athletic controls). The weighted overall mean for the prevalence of elevated $\mathrm{T} 1$ was $0 \%$, T2 was $0 \%$, LGE including hinge-point fibrosis was $8.5 \%$, LGE excluding hinge-point fibrosis was $0 \%$, PE not reported and $0 \%$ met the LLC for myocarditis. Clark et $a l^{34}$ reported no values for $\mathrm{T} 1$ and $\mathrm{T} 2$ but $24 \%(\mathrm{n}=10)$ athletic controls with hinge-point fibrosis.

\section{Echocardiography findings}

Study and demographic characteristics of studies using echocardiography as their main imaging modality with level of evidence are summarised in table 2. The median time interval from symptom onset to echocardiography was 27 (IQR 3-156) days. The most frequently reported echocardiography parameters were LVEF, TAPSE and PE. In seven studies comprising 2606 athletes and study controls, the weighted mean for LVEF was $50.8 \%$, TAPSE $19.9 \mathrm{~mm}$ and the prevalence of PE $0.6 \%$.

\section{Athletes}

In total, six studies reported findings in 2574 athletes (1677 student athletes, 879 professional athletes and 18 elite athletes), in which $36 \%$ were asymptomatic, $60 \%$ 


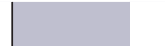

a

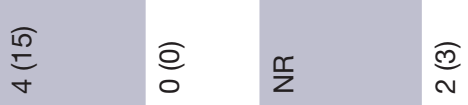

$\underset{N}{\stackrel{O}{\infty}}$

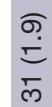

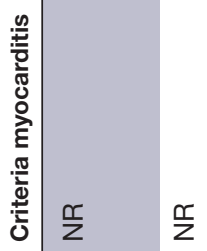

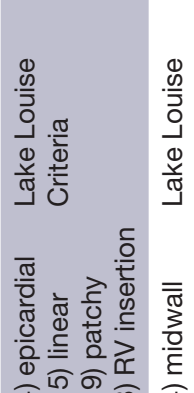

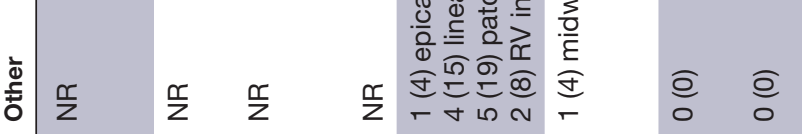

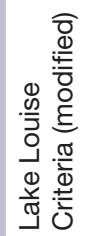

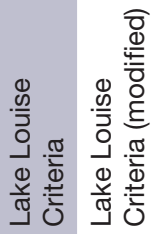

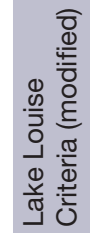

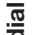

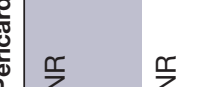

$\frac{\mathfrak{x}}{2} \cong$

$\frac{1}{z}$

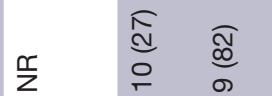

$\bar{\pi}$
$\frac{0}{0}$
$\frac{1}{\infty}$

完市

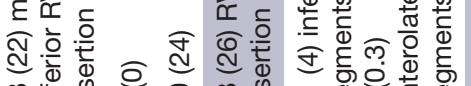

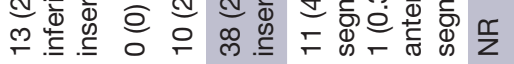

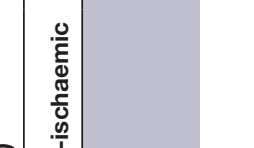

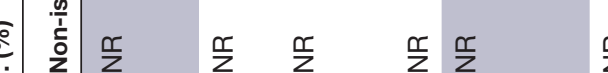

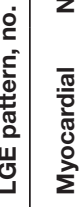

蓠

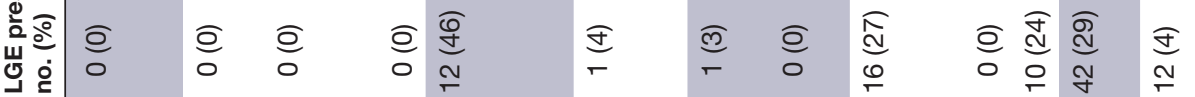

๖

$\stackrel{\circ}{\check{1}}$

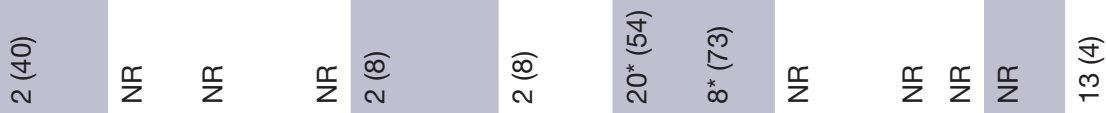

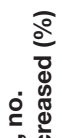

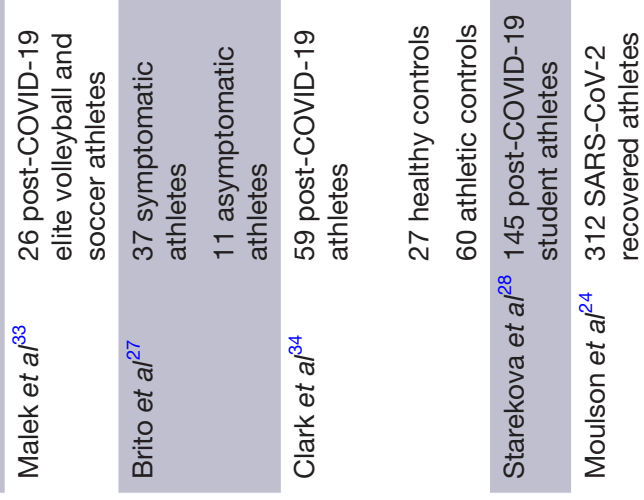

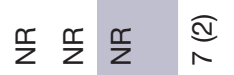




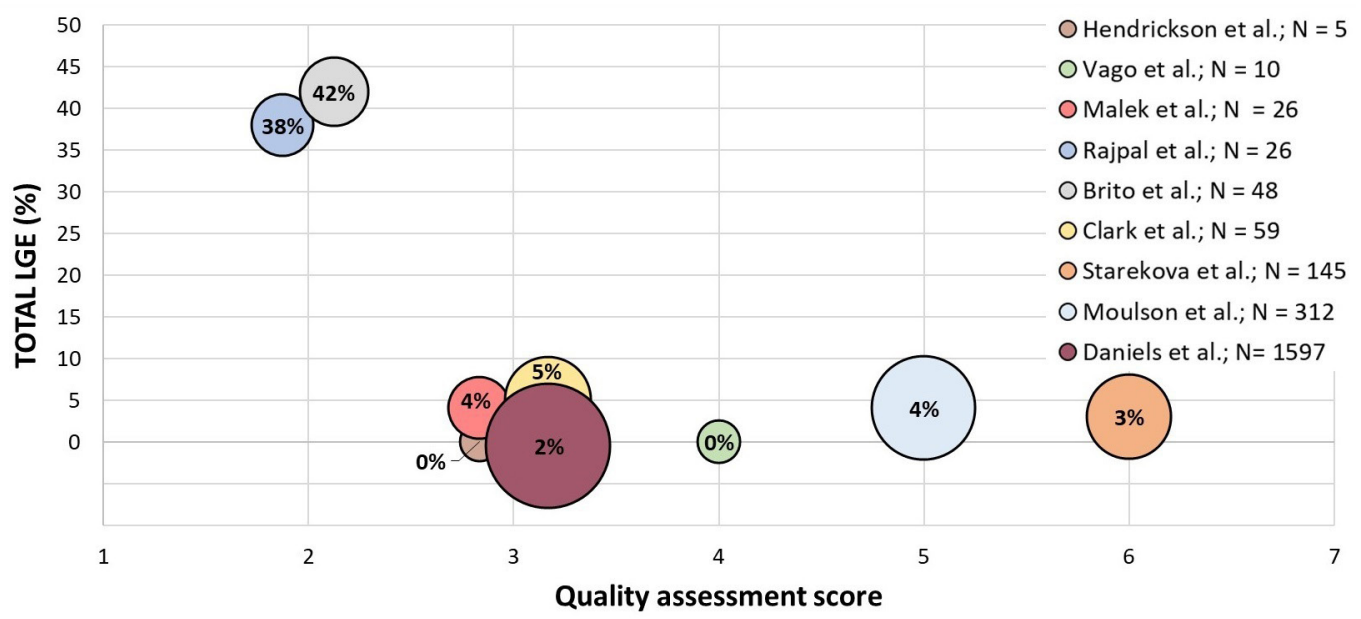

Figure 2 Total \% of late gadolinium enhancement (LGE) detected on CMR stratified by risk of bias based on the quality assessment score. Each circle represents a single study. The area of the sphere represents the size of the study population. CMR, cardiovascular magnetic resonance.

had mild symptoms, $4 \%$ had moderate symptoms and 0 had severe symptoms. The weighted mean for LVEF was $50.7 \%$, for TAPSE $19.5 \mathrm{~mm}$ and for the prevalence of PE $0.6 \%$. Brito et $a l^{27}$ reported no significant differences between asymptomatic and symptomatic student athletes for LVEF $(p=0.5)$ and PE $(p=0.5)$. They identified three so-called phenotypic student athlete groups: mild pericardial disease, pericardial disease and mild myocardial disease on CMR. In a hierarchical clustering analysis, these groups were associated with GLS $<-16 \%$ on echocardiography. Furthermore, Hendrickson $e t a l^{\beta 1}$ reported only a trace of PE in $4(2.9 \%)$ athletes, with further normal evaluations. Gervasi et $a l^{24}$ reported no significant differences between COVID-19 positive soccer players and COVID-19 negative soccer players for LVEF ( $p=0.55$ ) and TAPSE $(\mathrm{p}=0.69)$. In addition, Moulson $e t a l^{24}$ only reported echocardiography data in 21 athletes with pericardial/myocardial involvement. Only those athletes

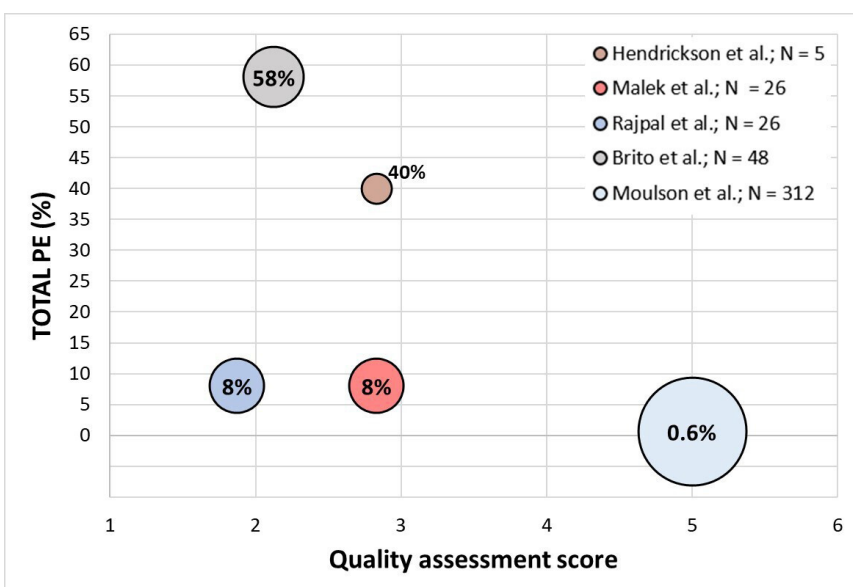

Figure 3 Total \% of pericardial effusion (PE) detected on CMR stratified by risk of bias based on the quality assessment score. Each circle represents a single study. The area of the sphere represents the size of the study population. PE, pericardial effusion. could therefore be included in the data synthesis, instead of the total echocardiography population $(n=2231)$. The study of Daniels $e t a l^{25}$ reported one athlete with symptoms of dyspnoea, with decreased LVEF (35\%-40\%) on echocardiography and CMR. Additionally, Martinez et $a l^{35}$ reported sixcases of pericarditis/myocarditis (confirmed on CMR) of which four had abnormal echocardiography findings ('small' (not further defined) PE, LVEF 50\%, new region wall abnormalities/preserved LVEF and dilated RV). Finally, Cavigli et $a l^{23}$ reported $\mathrm{PE}$ in three athletes with symptomatic SARS-CoV-2 infections, of which one confirmed myopericarditis and two cases of pericarditis.

Study controls (healthy and/or athletes)

In total two studies reported findings in 32 athletic controls. The weighted overall mean LVEF was $60.2 \%$, for TAPSE -20.5 and for the prevalence of PE $0 \%$. Gervasi $e t$ $a l$ did not report strain and $\mathrm{PE}$ values.

\section{Arrhythmias and SCA/SCD}

Within a setting of a non-systematic FU period of 113 days, the large cohort of Moulson et al $(\mathrm{n}=2820)$ demonstrated no instances of SCD, and only one (0.04\%) resuscitated SCA. The case of SCA had a prior CMR, performed 17 days after onset of symptoms of COVID-19, without findings suggestive of acute cardiac involvement. The authors conclude that the aetiology of this event was considered uncertain and likely unrelated to COVID19. No arrhythmia monitoring was performed during FU. Gervasi et al reported arrhythmia outcomes: one athlete $(5.6 \%)$ had ventricular premature beats (VPBs) during exercise and one athlete $(5.6 \%)$ had supraventricular premature beats (SVPBs) during exercise, which were not present at exercise testing prior to SARS-CoV-2 infection. Holter monitoring was only performed postrecovery and demonstrated rare or occasional, isolated SVPB and/or VBP in all athletes. In addition, Cavigli et al reported arrhythmia outcomes: 24-hour ambulatory ECG 
monitoring showed isolated VPBs and SVPBs in 53.3\% and $52.5 \%$, respectively, with no malignant arrhythmias. During CPET, neither VPBs nor ST segment or T wave abnormalities were found, except for an athlete showing isolated VPBs and couplets (RBBB configuration with wide QRS-complex) at peak exercise and immediately after exercise. In this athlete, echocardiography demonstrated the presence of mild pericardial effusion and CMR was found to be suggestive for myopericarditis.

\section{Troponin and cardiac abnormalities}

In total, 10 studies comprising 4171 athletes and study controls reported serum troponin T or I levels. In studies reporting CMR-documented cardiac abnormalities, the weighted mean of elevated troponin levels (according to individual study cut-offs) was $0.65 \%$ in athletes and $0 \%$ in controls. The weighted mean for elevated troponin levels in echocardiography studies (at time of echocardiography) was $0.25 \%$ in athletes and $0 \%$ in controls. Moulson et al reported an elevated troponin level in $0.9 \%$ of the entire cohort $(\mathrm{n}=2719)$ but did not report any association with CMR or echocardiography abnormalities. Gervasi $e t$ al and Clark et al did not report troponin levels in study controls. No study reported a clear relationship between elevated troponin levels and cardiac abnormalities on imaging investigations.

\section{DISCUSSION}

Our systematic review addressing cardiac abnormalities in athletes recovered from SARS-CoV-2 infection found marked heterogeneity in investigations, study populations and methodology. The majority of abnormal pericardial/myocardial findings were reported by studies performing CMR as part of their investigations. Overall, myocardial abnormalities on CMR such as myocardial LGE and confirmed myocarditis (LLC) was reported in $0 \%-15 \%$ of cases. Pericardial abnormalities such as PE and pericardial LGE were reported in $0 \%-57 \%$ (CMR) of cases. In the total population, myocarditis was reported on echocardiography and/or CMR in $0 \%-15 \%$, PE in $8 \%-58 \%$ and LGE in $0 \%-46 \%$. Importantly, our findings also demonstrate that lower quality studies tended to report considerably higher rates of abnormalities $(38 \%-$ $42 \%$ ). Studies with higher quality assessment scores showed a somewhat greater agreement in proportions of athletes with LGE $(0 \%-5 \%)$ after SARS-CoV-2 infection. As such, our review highlights that emergent, small, lowquality studies should be interpreted with caution and a need for prospective, large athlete cohort studies with a low risk of bias.

\section{Clinical implications}

This review demonstrates that the incidence of post-SARSCoV-2 myocardial abnormalities detected on CMR is low and that CMR is the most sensitive diagnostic modality to detect myocardial abnormalities in athletes. As such, using CMR as a screening modality to detect potential SARS-CoV-2 myocardial abnormalities warrants further investigation. Additionally, while no studies systematically performed extensive arrhythmia monitoring in individuals with clear myocardial abnormalities, these studies also report no clear cases of SCA/SCD. Therefore, until prospective studies have been published, systematic (arrhythmia) monitoring is recommended in athletes with myocardial abnormalities after SARS-CoV-2. Finally, and perhaps most relevant for sports physicians directly involved in RTS in athletes, troponin screenings outside of specific clinical contexts (ie, cardiac complaints or other diagnostic abnormalities) should not occupy a central place in return-to-play strategies.

FU CMR was reported in only one study (Daniels $e t$ al) and demonstrated complete reversibility in approximately $40 \%$ of individuals diagnosed with myocarditis. Two studies (Gervasi et al and Cavigli et al) investigated arrhythmia outcomes, with only a single athlete demonstrating couplets at peak effort and in early recovery; this athlete was consequently diagnosed with myopericarditis. One study reported a single instance of resuscitated SCA likely unrelated to COVID-19, with no abnormalities on a prior CMR performed after SARS-CoV-2 infection. No study reported a clear relationship between elevated troponin levels and cardiac abnormalities on imaging investigations. Based on the currently available data, the short-term risk for post-COVID-19 SCA/SCD due to arrhythmias caused by myocardial inflammation appears to be poorly investigated, yet reported numbers are low, and the propensity for SARS-CoV-2 pericardial and myocardial involvement variable.

Although physical activity is thought to negatively influence prognosis in athletes with pericarditis and myocarditis, ${ }^{5}$ the rate of myocarditis in athletes after SARS-CoV-2 infection appears low. Overall, athletes were asymptomatic $(36 \%)$ or had mild symptoms (59\%), which may be reflected in the low rates of myocardial injury. FU data in athletes who continue to perform sports after SARS-CoV-2 infection is needed to further elucidate a potential interaction between physical activity and myocardial involvement.

All CMR studies were cross-sectional or retrospective, and only a single study with a small sample size $(n=18)$ performed comparative echocardiography analyses with pre- SARS-CoV-2 infection imaging. While it seems plausible that athletes who fulfil the LLC for myocarditis shortly after infection with SARS-CoV-2 have SARS-CoV-2 myocardial involvement, a recent study in healthcare workers has questioned whether such abnormalities are more common in individuals recovered from SARS-CoV-2 infection when compared with an adequate control group. ${ }^{36}$ Therefore, caution is advised before attributing (isolated) cardiac abnormalities to SARS-CoV-2. Ideally, to rule out pre-existent abnormalities due to other causes, imaging studies in athletes with pre-existing CMRs should be performed, preferably in well-defined groups as opposed to all comers in imaging centres, with core-lab assessment of all imaging studies. Furthermore, 
considering the potential reversibility of pericardial/ myocardial abnormalities reported by Daniels et al, the extent, and time course and long-term reversibility has yet determined.

Echocardiography findings in our study population did not demonstrate a high prevalence of major cardiac abnormalities, or significant differences compared with in-study controls. Of note, only Brito et al reported a high prevalence of pericardial LGE in athletes. Surprisingly, after multimodality adjudication, they demonstrated an association between GLS $<-16 \%$ on echocardiography and pericardial/myocardial abnormalities on CMR. First, as GLS is mainly a myocardial parameter, this association hints at possible pericardial/myocardial involvement, not detectable on CMR. Second, while GLS could potentially be a promising tool to identify COVID-19 associated pericardial/myocardial involvement, this finding has not yet been replicated in other studies, and hence such a strategy should not be implemented in routine care for post-COVID-19 athletes. Second, Martinez et $a l^{85}$ reported four (of six) cases with abnormal echocardiography (with varying findings) and confirmed pericarditis/myocarditis on CMR. However, they only performed CMRs in athletes with a suspicion of pericardial/myocardial involvement after return-to-play screening, and it is unclear whether athletes without conventional screening abnormalities also had CMR abnormalities. Therefore, according to our findings, screening echocardiography should be used with caution to perform rule-outs for subclinical myocarditis.

Several groups have speculated on causes for SCA/SCD related to COVID-19, such as possible proarrhythmic genetic predisposition or comorbidities that potentially increase the risk of drug-induced and hypoxia-induced ventricular arrhythmias/SCD. ${ }^{37} 38$ Yet, while the studies identified by our systematic review extensively investigated and reported myocardial abnormalities as found using CMR, none of these studies performed a structured investigation of potential arrhythmias. One study (Gervasi $e t a l$ ) found no clinically important arrhythmias in 18 football players. However, in addition to the small sample size, this study did not include CMR to document myocardial abnormalities. One study (Moulson et al) reported a resuscitated SCA (likely unrelated to SARSCoV-2) with no abnormal findings on prior CMR after SARS-CoV-2 infection. Hence, these findings cannot readily be extrapolated to athletes with potential or suspected myocardial involvement after COVID-19.

The majority of the studies included in our systematic review determined troponin levels after recovery from COVID-19. Of import, no single paper reported a statistically significant association between elevated serum troponin levels and CMR or echocardiography abnormalities. Therefore, the use of serum troponin after recovery to predict SARS-CoV-2 myocardial involvement remains questionable, with the potential for both false negative and false positive outcomes. According to our findings, troponin testing should not occupy a central place in return-to-play algorithms, and only be applied in appropriate (expert) clinical settings.

\section{Strengths and limitations}

There are several strengths to our study. First, we performed a systematic search through multiple databases, including articles ahead-of-print. Second, data extraction was rigorously performed to report each diagnostic finding. Third, we were able to stratify and weight main findings according to athlete level and recovery location (in-hospital or/and at home). Finally, all studies underwent extensive quality assessment, enabling us to stratify central findings according to study quality.

A number of aspects of our systematic review warrant consideration. First, the majority of the studies included in our review were found to have risk of bias. However, the scarcity of data on this topic in an emerging pandemic makes this a currently unavoidable bias. Second, we did not include case reports in our systematic review, as these included non-athletes with high rates of comorbidities, and because such reports have an intrinsic selection bias. Third, the data across the identified studies was heterogeneous, which limits generalisability. Fourth, the time interval between symptoms onset to cardiac imaging was too variable to allow for a cumulative analysis. Finally, we focused on late complications including late arrhythmias (ie, post-COVID-19) and not on the arrhythmia burden during an active SARS-CoV-2 infection.

\section{CONCLUSION}

Our systematic review on athletes recovered from COVID-19 confirms that infection with SARS-CoV-2 is associated with an overall low risk of pericardial/ myocardial involvement, arrhythmias and SCA/SCD. Individual studies show a highly variable degree of pericardial and myocardial abnormalities on CMR, which is associated with study quality. CMR appears to be more sensitive to detect (potentially relevant and reversible) post-COVID-19 pericardial/myocardial abnormalities. The extent, time course and potential reversibility of SARS-CoV-2 cardiac abnormalities remain inconclusive. Routine serum troponin screenings after recovery appear unreliable in identifying individuals at risk for such involvement. Prospective studies, preferentially with pre-SARS-CoV-2 imaging (CMR), in larger, well-defined populations, including longer term FU and arrhythmia monitoring, are urgently needed.

Contributors Each of the authors confirm that this manuscript has not been previously published and is not currently under consideration by any other journal. Furthermore, all of the authors have approved the contents of this paper and have agreed the BJM Open Sport \& Exercise Medicine policies. Each named author has substantially contributed to drafting this manuscript. Additionally, to the best of our knowledge, the named authors have no conflict of interest, financial or otherwise.

Funding HJ has received funding from the Amsterdam Movement Sciences (P1A210AMC2018) and the National Olympic Committee \& National Sports Federation.

Competing interests None declared.

Patient consent for publication Not applicable. 
Provenance and peer review Not commissioned; externally peer reviewed.

Open access This is an open access article distributed in accordance with the Creative Commons Attribution Non Commercial (CC BY-NC 4.0) license, which permits others to distribute, remix, adapt, build upon this work non-commercially, and license their derivative works on different terms, provided the original work is properly cited, appropriate credit is given, any changes made indicated, and the use is non-commercial. See: http://creativecommons.org/licenses/by-nc/4.0/.

\section{ORCID iDs}

Juliette C van Hattum http://orcid.org/0000-0003-0168-7783

Harald T Jorstad http://orcid.org/0000-0003-3617-3256

\section{REFERENCES}

1 World Health Organization. WHO Director-General's opening remarks at the media briefing on COVID-19 - 11 March 2020. Available: https://www.who.int/director-general/speeches/detail/who-directorgeneral-s-opening-remarks-at-the-media-briefing-on-covid-19-11march-2020

2 Cruz Rodriguez JB, Lange RA, Mukherjee D. Gamut of cardiac manifestations and complications of COVID-19: a contemporary review. J Investig Med 2020;68:1334-40.

3 Kiel RJ, Smith FE, Chason J, et al. Coxsackievirus B3 myocarditis in $\mathrm{C} 3 \mathrm{H} / \mathrm{HeJ}$ mice: description of an inbred model and the effect of exercise on virulence. Eur J Epidemiol 1989;5:348-50.

4 Ilbäck NG, Fohlman J, Friman G. Exercise in Coxsackie B3 myocarditis: effects on heart lymphocyte subpopulations and the inflammatory reaction. Am Heart J 1989;117:1298-302.

5 Phillips M, Robinowitz M, Higgins JR, et al. Sudden cardiac death in air force recruits. A 20-year review. JAMA 1986;256:2696-9.

6 Metzl JD, McElheny K, Robinson JN, et al. Considerations for return to exercise following mild-to-moderate COVID-19 in the recreational athlete. Hss J 2020;16:102-7.

7 Elliott N, Martin R, Heron N, et al. Infographic. graduated return to play guidance following COVID-19 infection. Br J Sports Med 2020;54:1174-5.

8 Verwoert GC, de Vries ST, Bijsterveld N, et al. Return to sports after COVID-19: a position paper from the Dutch Sports Cardiology Section of the Netherlands Society of Cardiology. Neth Heart $J$ 2020;28:391-5.

$9 \mathrm{Kim}$ JH, Levine BD, Phelan D, et al. Coronavirus disease 2019 and the athletic heart: emerging perspectives on pathology, risks, and return to play. JAMA Cardiol 2021;6:219-27.

10 Huang C, Wang Y, Li X, et al. Clinical features of patients infected with 2019 novel coronavirus in Wuhan, China. Lancet 2020;395:497-506.

11 Zhou F, Yu T, Du R, et al. Clinical course and risk factors for mortality of adult inpatients with COVID-19 in Wuhan, China: a retrospective cohort study. Lancet 2020;395:1054-62.

12 Lippi G, Lavie CJ, Sanchis-Gomar F. Cardiac troponin I in patients with coronavirus disease 2019 (COVID-19): evidence from a metaanalysis. Prog Cardiovasc Dis 2020;63:390-1.

13 Wang D, Hu B, Hu C, et al. Clinical characteristics of 138 hospitalized patients with 2019 novel coronavirus-infected pneumonia in Wuhan, China. JAMA 2020;323:1061-9.

14 Imazio M, Klingel K, Kindermann I, et al. COVID-19 pandemic and troponin: indirect myocardial injury, myocardial inflammation or myocarditis? Heart 2020;106:1127-31.

$15 \mathrm{Kim}$ JH, Levine BD, Phelan D, et al. Coronavirus disease 2019 and the athletic heart: emerging perspectives on pathology, risks, and return to play. JAMA Cardiol 2021;6:219-227.

16 Phelan D, Kim JH, Elliott MD, et al. Screening of potential cardiac involvement in competitive athletes recovering from COVID-19: an expert consensus statement. JACC Cardiovasc Imaging 2020;13:2635-52

17 Moher D, Liberati A, Tetzlaff J, et al. Preferred reporting items for systematic reviews and meta-analyses: the PRISMA statement. $J$ Clin Epidemiol 2009;62:1006-12.
18 Pelliccia A, Sharma S, Gati S, et al. 2020 ESC guidelines on sports cardiology and exercise in patients with cardiovascular disease. Eur Heart J 2021;42:17-96. Erratum in: Eur Heart J. 2021 Feb $1 ; 42(5): 548-549$.

19 van Hattum JC, Verwijs SM, Rienks R, et al. The Netherlands Sports Cardiology Map: a step towards sports cardiology network medicine for patient and athlete care. Neth Heart J 2021;29:129-134

20 Field AP. Discovering statistics using SPSS: (and sex and drugs and rock ' $n$ ' roll. Third edition. Los Angeles [i.e. Thousand Oaks, Calif; London: Printed Book. SAGE Publications, 2009.

21 Moola S, Munn Z, Tufanaru C. Chapter 7: Systematic reviews of etiology and risk. In: Aromataris E, Munn Z, eds. JBI manual for evidence synthesis. JBI, 2020. https://synthesismanual.jbi.global

22 Gervasi SF, Pengue L, Damato L, et al. Is extensive cardiopulmonary screening useful in athletes with previous asymptomatic or mild SARS-CoV-2 infection? Br J Sports Med 2021;55:54-61.

23 Cavigli L, Frascaro F, Turchini F, et al. A prospective study on the consequences of SARS-CoV-2 infection on the heart of young adult competitive athletes: implications for a safe return-to-play. Int $J$ Cardiol 2021;336:130-6.

24 Moulson N, Petek BJ, Drezner JA, et al. SARS-CoV-2 cardiac involvement in young competitive athletes. Circulation 2021:144:256-66.

25 Daniels CJ, Rajpal S, Greenshields JT, et al. Prevalence of clinical and subclinical myocarditis in competitive athletes with recent SARS-CoV-2 infection: results from the big ten COVID-19 cardiac registry. JAMA Cardiol 2021;6:1078-1087.

26 Rajpal S, Tong MS, Borchers J, et al. Cardiovascular magnetic resonance findings in competitive athletes recovering from COVID-19 infection. JAMA Cardiol 2021;6:116-8.

27 Brito D, Meester S, Yanamala N, et al. High prevalence of pericardial involvement in college student athletes recovering from COVID-19. JACC Cardiovasc Imaging 2021;14:541-55.

28 Starekova J, Bluemke DA, Bradham WS, et al. Evaluation for myocarditis in competitive student athletes recovering from coronavirus disease 2019 with cardiac magnetic resonance imaging. JAMA Cardiol 2021;6:1-6.

29 Friedrich MG, Sechtem U, Schulz-Menger J. International consensus group on cardiovascular magnetic resonance in myocarditis. cardiovascular magnetic resonance in myocarditis: a JACC white paper. J Am Coll Cardiol 2009;53:1475-87.

30 Ferreira VM, Schulz-Menger J, Holmvang G, et al. Cardiovascular Magnetic Resonance in Nonischemic Myocardial Inflammation. J Am Coll Cardiol 2018;72:3158-76

31 Hendrickson BS, Stephens RE, Chang JV, et al. Cardiovascular evaluation after COVID-19 in 137 collegiate athletes: results of an Algorithm-Guided screening. Circulation 2021;143:1926-8.

32 Vago H, Szabo L, Dohy Z, et al. Cardiac magnetic resonance findings in patients recovered from COVID-19: initial experiences in elite athletes. JACC Cardiovasc Imaging 2021;14:2-3.

33 Małek Łukasz A, Marczak M, Miłosz-Wieczorek B, et al. Cardiac involvement in consecutive elite athletes recovered from Covid-19: A magnetic resonance study. J Magn Reson Imaging 2021;53:1723-9.

34 Clark DE, Parikh A, Dendy JM. COVID-19 myocardial pathology evaluation in athletes with cardiac magnetic resonance (compete CMR). Circulation 2020.

35 Martinez MW, Tucker AM, Bloom OJ, et al. Prevalence of inflammatory heart disease among professional athletes with prior COVID-19 infection who received systematic Return-to-Play cardiac screening. JAMA Cardiol 2021;6:745.

36 Joy G, Artico J, Kurdi H, et al. Prospective Case-Control Study of Cardiovascular Abnormalities 6 Months Following Mild COVID-19 in Healthcare Workers. JACC Cardiovasc Imaging 2021. doi:10.1016/j.jcmg.2021.04.011. [Epub ahead of print: 05 May 2021].

37 Giudicessi JR, Roden DM, Wilde AAM, et al. Genetic susceptibility for COVID-19-associated sudden cardiac death in African Americans. Heart Rhythm 2020;17:1487-92.

38 Wu C-I, Postema PG, Arbelo E, et al. SARS-CoV-2, COVID-19, and inherited arrhythmia syndromes. Heart Rhythm 2020;17:1456-62. 